\title{
Chromospheric activity of stars with planets
}

\author{
B. L. Canto Martins, M. L. das Chagas, S. Alves, I. C. Leão, L. P. de Souza Neto, and J. R. de Medeiros
}

Departamento de Física, Universidade Federal do Rio Grande do Norte, Natal, Brazil

e-mail: brunocanto@dfte.ufrn.br

Received 30 June 2010 / Accepted 25 March 2011

\section{ABSTRACT}

\begin{abstract}
Context. Signatures of chromospheric activity enhancement have been found for a dozen stars, pointing to a possible star-planet interaction. Nevertheless in the coronal activity regime, there is no conclusive observational evidence of such an interaction. Does star-planet interaction manifest itself only for a few particular cases, without having a major effect on stars with planets in general? Aims. We aim to add additional observational constraints to support or reject the major effects of star-planet interactions in stellar activity, based on Ca II chromospheric emission flux.

Methods. We performed a statistical analysis of Ca II emission flux of stars with planets, as well as a comparison between Ca II and $\mathrm{X}$-ray emission fluxes, searching for dependencies on planetary parameters.

Results. In the present sample of stars with planets, there are no significant correlations between chromospheric activity indicator $\log \left(R_{\mathrm{HK}}^{\prime}\right)$ and planetary parameters. Furthermore, the distribution of the chromospheric activity indicator for stars without planets is indistinguishable from the one with planets.
\end{abstract}

Key words. planet-star interactions - stars: activity - stars: chromospheres - stars: coronae - stars: statistics

\section{Introduction}

Since the pioneering discovery by Mayor \& Queloz (1995) a little more than a decade ago - of a planet orbiting the star $51 \mathrm{Peg}$, more than 450 other exoplanets have been found at the time of writing (e.g., Schneider 2010). The discovered planets have masses ranging from 4 Earth masses to 11 Jupiter masses. They can be found at distances of several AU or close to the parent star, with orbital periods ranging from a few days to a few years. High eccentricity is a common parameter connecting these lanets (e.g., Marcy et al. 2001).

These discoveries have inspired intensive studies of the physical properties of the planets and of their parent stars, including a possible star-planet interaction. Indeed, in an analogy to binary stars, which show a higher activity level compared to single stars, at very close distances, one might also expect planets to play a role in the level of activity of their host stars. Nevertheless, observational data, still at a very informative level, demonstrate that star-planet interaction appears to be more complex than the widely accepted star-star interaction in close binary systems. For instance, Shkolnik et al. (2003, 2008) report a planet-induced chromospheric activity on two stars with planets, HD 179949 and $v$ And, apparent from the night-to-night modulation of the CaII $\mathrm{H}$ and $\mathrm{K}$ chromospheric emission phased with the hot Jupiter's orbit. In addition, Kashyap et al. (2008) claim that stars with close-in giant planets are on average more X-ray active than those with planets that are more distant, an observational result consistent with the hypothesis that giant planets in close proximity to the parent stars could influence stellar magnetic activity.

By contrast, Poppenhaeger et al. (2010) find no significant correlations between X-ray luminosity and planetary parameters, suggesting no major average activity enhancement in the corona of stars with planets. Indeed, these authors find no additional detectable effects in coronal X-ray luminosity that could be associated to coronal manifestations of star-planet interaction. According to Poppenhaeger et al. (2010), any trends in the X-ray luminosity versus planet orbital parameters seem to be dominated by selection effects. In addition, Fares et al. (2010) reports no clear evidence of star-planet magnetospheric interactions in HD 189733. In this context, let us recall that, more recently from a comparison of the distribution of the rotation of stars with and without detected planets, Alves et al. (2010) have shown that the $v \sin i$ distribution for these two families of stars is drawn from the same population distribution function.

Because the observational basis of stellar activity enhancement due to star-planet interaction has clearly not yet been established, in the present study we report a statistical analysis of stellar CaII chromospheric emission flux for a sample of 74 stars with planets, as described in the following sections. For a more solid analysis of the behavior of chromospheric and coronal activity of stars with planets, it is essential to conduct a comparative analysis of stars without detected planets. This is one of the major goals of the current study. In this context, we also analyzed the CaII chromospheric emission flux of a comparison sample of 26 stars without detected planets. The paper is organized as follows. In Sect. 2 we present the characteristics of the working samples, in Sect. 3 we describe our findings with a brief discussion, and finally we outline our conclusions in Sect. 4.

\section{Stellar working sample and data}

Different methods, such as radial velocity measurements (Butler et al. 1996; de Medeiros et al. 2009) and photometric light curves (Konacki et al. 2003, 2004), have been used to discover new planetary systems around other stars than the Sun. Aiming to make our sample homogeneous, we only use stars that had their planets discovered with radial velocity method.

The present stellar working sample consists of selected objects from the base of extrasolar planets maintained by 
Jean Schneider (Schneider 2010), updated on October 6, 2009, when there were 235 planets cataloged by the method in variation in the radial velocity (RV). We checked which of these stars presented Ca II emission flux detected in the catalog of Wright et al. (2004). After this selection, we filtered the complete stellar sample to obtain a subset of main-sequence stars that are within $20 \mathrm{pc}$ in the solar neighborhood. Our final working sample consists of 74 stars with planets with spectral types in the F-G-K interval, as presented in Table 1.

The chromospheric activity indicator, $\log \left(R_{\mathrm{HK}}^{\prime}\right)$, for all stars was computed from the $\mathrm{Ca}$ II $\mathrm{H}$ and $\mathrm{K}$ line-core emission index listed by Wright et al. (2004), following the procedure of converting emission index $S$ to the flux at the stellar surface proposed by Noyes et al. (1984). To diagnose coronal activity of the stars forming the above-mentioned stellar sample, we used the coronal activity indicator, $\log \left(\frac{L_{X}}{L_{\mathrm{bol}}}\right)$, that was computed using the X-ray fluxes listed by Kashyap et al. (2008) and Poppenhaeger et al. (2010). Readers are referred to these works for a discussion of the observational procedures, data reduction, and error analysis.

Stellar luminosities were determined as follows. First, apparent visual magnitudes $\left(m_{\mathrm{v}}\right)$ and trigonometric parallaxes, both taken from the HIPPARCOS catalogue (ESA 1997), were combined to yield the absolute visual magnitude $\left(M_{\mathrm{v}}\right)$. Bolometric correction $(B C)$, computed from Flower (1996) calibration, was applied to obtaining the bolometric magnitude, which was finally converted into stellar luminosity. The effective temperature was computed using Flower (1996) $(B-V)$ versus $T_{\text {eff }}$ calibration. The stellar and planetary parameters, as well as chromospheric and coronal activity indicators for the entire sample of stars with planets, are listed in Table 1.

For comparative purposes, we took a sample of stars not known to have any planetary-mass companions. This new sample was taken from Schmitt et al. (1997), as done by Poppenhaeger et al. (2010), and is composed of 26 F-G-K type dwarf stars with all stars having Ca II emission flux measured by Wright et al. (2004) and X-ray fluxes detected by Schmitt (1997). The chromospheric and coronal activity indicators were calculated as before for the sample of stars with planets. However, we should be cautious about this sample, which derives from a list of stars that are surveyed for planets, but for which none have yet been found. This certainly does not mean that such stars have no planetary companions whatsoever. In fact, they might host planets with very low mass and/or a long orbital period that are more difficult to detect with radial velocity surveys. Stellar properties and chromospheric and coronal activity indicators for stars without detected planets are given in Table 2.

\section{Results and discussion}

The aim of this work is to point out additional observational constraints that support or reject major effects of star-planet interaction in stellar activity, based on CaII chromospheric and X-ray emission fluxes. To this end, we dedicated most of our efforts to identifying qualitative trends between $\mathrm{CaII}$ and $\mathrm{X}$-ray fluxes and planetary parameters. We chose $\log \left(R_{\mathrm{HK}}^{\prime}\right)$ and $\log \left(\frac{L_{\mathrm{X}}}{L_{\mathrm{bol}}}\right)$ as indicators of chromospheric and coronal activity, respectively, because they are independent of stellar radius-induced effects. Indeed, it is expected that any planet-induced activity changes should therefore be more evident in $\log \left(\frac{L_{\mathrm{X}}}{L_{\mathrm{bol}}}\right)$ (e.g.: Poppenhaeger et al. 2010). In the context of any planet-induced chromospheric activity, one should also expect more evident changes in $\log \left(R_{\mathrm{HK}}^{\prime}\right)$ for such an aspect.

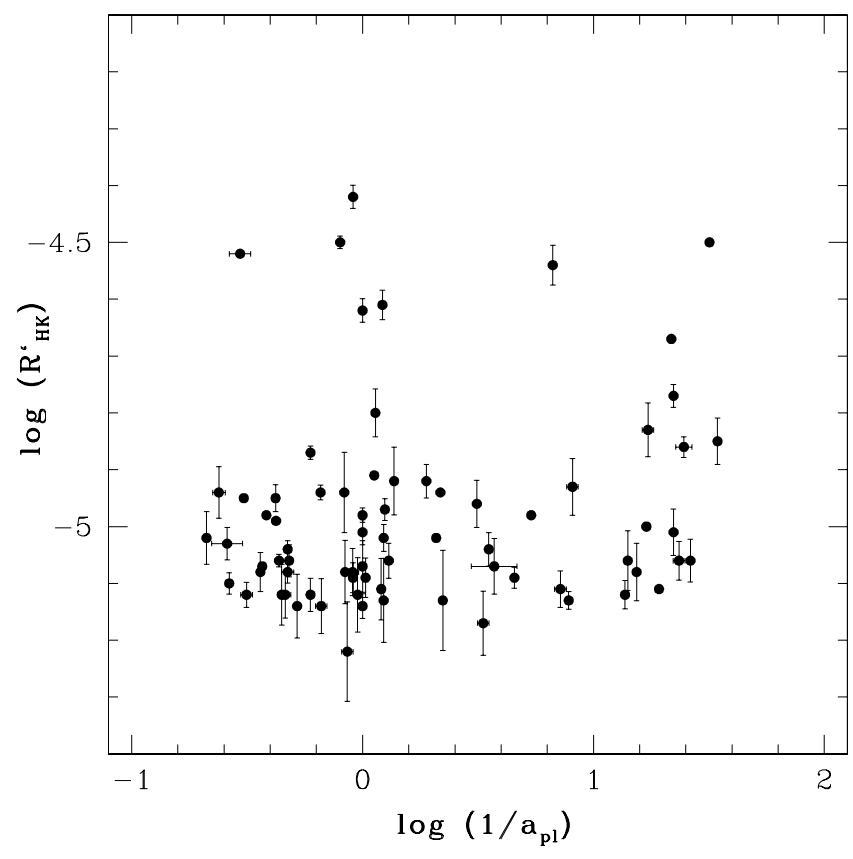

Fig. 1. Chromospheric activity indicator $\log \left(R_{\mathrm{HK}}^{\prime}\right)$ as a function of the planetary semi-major axis $\log \left(1 / a_{\mathrm{pl}}\right)$ for our sample of stars with planets.

Following the same strategy as used by Poppenhaeger et al. (2010) in their analysis of the coronal X-ray emission behavior in stars with planets, we show the distribution of the chromospheric activity indicator $\log \left(R_{\mathrm{HK}}^{\prime}\right)$ of stars with planets as a function of planetary distance $\log \left(1 / a_{\mathrm{pl}}\right)$ (Fig. 1). A close comparison of the distribution of $\log \left(R_{\mathrm{HK}}^{\prime}\right)$ versus $\log \left(1 / a_{\mathrm{pl}}\right)$ with the distribution of the coronal activity indicator $\log \left(\frac{L_{\mathrm{X}}}{L_{\mathrm{bol}}}\right)$ versus $\log \left(1 / a_{\mathrm{pl}}\right)$, illustrated in Fig. 5 of Poppenhaeger et al. (2010), shows that, at least qualitatively, the behavior of the chromospheric activity indicator appears to be comparable to that of the coronal activity indicator. The same aspect can be seen in Fig. 2 where we show the distribution of the chromospheric activity indicator $\log \left(R_{\mathrm{HK}}^{\prime}\right)$ of stars with planets as a function of the product of the planetary mass with the reciprocal distance $\log \left(1 / a_{\mathrm{pl}}\right)$.

Still following the strategy proposed by Poppenhaeger et al. (2010), we also carried out a statistical analysis of both $\log \left(R_{\mathrm{HK}}^{\prime}\right)$ and $\log \left(\frac{L_{\mathrm{X}}}{L_{\mathrm{bol}}}\right)$ activity indicators of stars with close-in planets, namely planets with $a_{\mathrm{pl}} \leq 0.20 \mathrm{AU}$, searching for correlations with planetary mass and semi-major axis. Table 3 shows the result of such an analysis, with the Spearman's $\rho$ rank correlation for various combinations of stellar quantities and planetary parameters, for two samples. The first is composed of 19 stars with X-ray lumisonity from Kashyap et al. (2008), and the second is composed of 13 stars with X-ray luminosities from Poppenhaeger et al. (2010). The first interesting aspect of this analysis is a possible anticorrelation between the chromospheric activity indicator and the planetary semi-major axis in stars hosting planets with $a_{\mathrm{pl}} \leq 0.20 \mathrm{AU}$ for both samples. When we consider the entire sample of stars, we observe an absence of any significant correlation between these two parameters, without any distinction of $a_{\mathrm{pl}}$ values.

Two possible correlations are found in the present analysis: one of planetary mass $\log \left(M_{\mathrm{pl}}\right)$ with $\log \left(R_{\mathrm{HK}}^{\prime}\right)$ and the other of products of the planetary mass with the reciprocal distance $\log \left(1 / a_{\mathrm{pl}} \times M_{\mathrm{pl}}\right)$ with $\log \left(R_{\mathrm{HK}}^{\prime}\right)$. Stars with giant and close-in planets exhibit higher $\log \left(R_{\mathrm{HK}}^{\prime}\right)$ values than those with 
B. L. Canto Martins et al.: On the chromospheric activity of stars with planets

Table 1. Stellar and planetary parameters and chromospheric and coronal activity indicators of stars with planets.

\begin{tabular}{|c|c|c|c|c|c|c|c|}
\hline Star & ST & $(B-V)$ & $a_{\mathrm{pl}}$ & $M_{\mathrm{pl}}$ & $\log \left(R_{\mathrm{HK}}^{\prime}\right)$ & $\log \left(\frac{L_{\mathrm{X}}}{L_{\mathrm{bol}}}\right)^{a}$ & $\log \left(\frac{L_{\mathrm{X}}}{L_{\mathrm{bol}}}\right)^{b}$ \\
\hline HD 3651 & K0V & $0.850 \pm 0.009$ & 0.284 & 0.200 & $-5.04 \pm 0.03$ & $-6.07 \pm 0.08$ & $-6.07 \pm 0.23$ \\
\hline HD 4203 & G5 & $0.771 \pm 0.021$ & $1.164 \pm 0.07$ & $2.070 \pm 0.18$ & $-5.22 \pm 0.09$ & $-5.18 \pm 0.22$ & \\
\hline HD 4208 & G5V & $0.664 \pm 0.004$ & 0.800 & 1.700 & $-4.97 \pm 0.02$ & -5.12 & \\
\hline HD 7924 & K0 & $0.826 \pm 0.006$ & 0.029 & 0.057 & $-4.85 \pm 0.04$ & & $-5.71 \pm 0.29$ \\
\hline HD 8574 & F8 & $0.577 \pm 0.011$ & 0.770 & 2.110 & $-5.06 \pm 0.03$ & -5.00 & \\
\hline HD 10697 & G5IV & $0.720 \pm 0.009$ & $2.160 \pm 0.12$ & $6.380 \pm 0.53$ & $-5.12 \pm 0.04$ & $-5.83 \pm 0.17$ & \\
\hline HD 12661 & K0 & $0.710 \pm 0.015$ & 0.830 & 2.300 & $-5.11 \pm 0.05$ & -5.44 & \\
\hline HD 16141 & G5IV & $0.670 \pm 0.004$ & 0.350 & $0.215 \pm 0.03$ & $-5.14 \pm 0.02$ & -5.32 & \\
\hline HD 19994 & F8V & $0.575 \pm 0.005$ & 1.680 & 1.420 & $-4.87 \pm 0.01$ & -6.04 & $-6.01 \pm 0.28$ \\
\hline HD 20367 & G0 & $0.574 \pm 0.008$ & 1.250 & 1.070 & $-4.50 \pm 0.01$ & $-4.54 \pm 0.03$ & $-4.42 \pm 0.12$ \\
\hline HD 23596 & F8 & $0.634 \pm 0.009$ & 2.720 & 7.190 & -5.07 & & -5.34 \\
\hline HD 30562 & F8V & $0.631 \pm 0.003$ & $2.300 \pm 0.02$ & $1.290 \pm 0.08$ & $-5.06 \pm 0.01$ & & -7.08 \\
\hline HD 37124 & G4IV-V & $0.667 \pm 0.008$ & $0.529 \pm 0.00$ & $0.640 \pm 0.05$ & $-4.92 \pm 0.03$ & -5.15 & \\
\hline HD 40979 & F8 & $0.573 \pm 0.007$ & 0.811 & $3.280 \pm 3.00$ & $-4.62 \pm 0.02$ & -5.51 & \\
\hline HD 45350 & G5 & $0.740 \pm 0.015$ & $1.920 \pm 0.07$ & $1.790 \pm 0.14$ & $-5.14 \pm 0.06$ & -5.11 & \\
\hline HD 46375 & K1IV & $0.860 \pm 0.000$ & 0.041 & $0.249 \pm 0.03$ & $-4.98 \pm 0.01$ & $-6.02 \pm 0.08$ & \\
\hline HD 49674 & G0 & $0.729 \pm 0.015$ & $0.058 \pm 0.00$ & $0.115 \pm 0.02$ & $-4.83 \pm 0.05$ & -5.02 & \\
\hline HD 50499 & G1V & $0.614 \pm 0.008$ & $3.860 \pm 0.60$ & $1.710 \pm 0.20$ & $-5.03 \pm 0.03$ & -4.98 & \\
\hline HD 50554 & F8 & $0.582 \pm 0.008$ & 2.380 & 4.900 & $-4.95 \pm 0.02$ & -6.81 & \\
\hline HD 52265 & G0III-IV & $0.572 \pm 0.003$ & 0.490 & $1.050 \pm 0.03$ & $-5.01 \pm 0.02$ & -5.60 & $-6.65 \pm 0.17$ \\
\hline HD 66428 & G5 & $0.715 \pm 0.002$ & $3.180 \pm 0.19$ & $2.820 \pm 0.03$ & $-5.12 \pm 0.02$ & -4.87 & \\
\hline HD 68988 & G0 & $0.652 \pm 0.015$ & 0.071 & 1.900 & $-5.06 \pm 0.05$ & -4.93 & \\
\hline HD 69830 & $\mathrm{~K} 0 \mathrm{~V}$ & $0.754 \pm 0.009$ & 0.186 & 0.038 & -4.98 & $-5.89 \pm 0.15$ & $-5.89 \pm 0.30$ \\
\hline HD 72659 & G0 & $0.612 \pm 0.015$ & $4.740 \pm 0.08$ & $3.150 \pm 0.01$ & $-5.02 \pm 0.05$ & -5.16 & \\
\hline HD 74156 & G0 & $0.585 \pm 0.014$ & 0.294 & 1.880 & $-5.07 \pm 0.05$ & -5.26 & \\
\hline HD 80606 & G5 & $0.765 \pm 0.025$ & $0.449 \pm 0.01$ & $3.940 \pm 0.11$ & $-5.13 \pm 0.09$ & -4.82 & \\
\hline HD 82943 & G0 & $0.623 \pm 0.001$ & 0.730 & 0.880 & $-4.92 \pm 0.06$ & -5.76 & -5.76 \\
\hline HD 83443 & $\mathrm{~K} 0 \mathrm{~V}$ & $0.811 \pm 0.003$ & $0.041 \pm 0.00$ & $0.400 \pm 0.03$ & $-4.86 \pm 0.02$ & -4.96 & \\
\hline HD 89307 & G0V & $0.594 \pm 0.003$ & $3.270 \pm 0.07$ & $1.780 \pm 0.13$ & -4.95 & -5.60 & \\
\hline HD 89744 & F7V & $0.531 \pm 0.003$ & 0.890 & 7.990 & -4.91 & $-6.31 \pm 0.07$ & \\
\hline HD 92788 & G5 & $0.694 \pm 0.005$ & 0.970 & 3.860 & $-5.09 \pm 0.03$ & -5.53 & \\
\hline HD 99109 & K0 & $0.874 \pm 0.002$ & $1.105 \pm 0.07$ & $0.502 \pm 0.07$ & $-5.08 \pm 0.04$ & -5.13 & \\
\hline HD 99492 & $\mathrm{~K} 2 \mathrm{~V}$ & $1.002 \pm 0.012$ & $0.123 \pm 0.01$ & $0.109 \pm 0.01$ & $-4.93 \pm 0.05$ & $-5.55 \pm 0.18$ & $-6.13 \pm 0.16$ \\
\hline HD 106252 & G0 & $0.635 \pm 0.007$ & 2.610 & 6.810 & -4.98 & -6.03 & \\
\hline HD 107148 & G5 & $0.707 \pm 0.013$ & $0.269 \pm 0.06$ & $0.210 \pm 0.04$ & $-5.07 \pm 0.05$ & -5.35 & \\
\hline HD 108874 & G5 & $0.738 \pm 0.018$ & $1.051 \pm 0.02$ & $1.360 \pm 0.13$ & $-5.12 \pm 0.07$ & -5.14 & \\
\hline HD 114729 & G0V & $0.591 \pm 0.008$ & 2.080 & 0.820 & $-5.06 \pm 0.03$ & -5.66 & \\
\hline HD 114783 & K0 & $0.930 \pm 0.013$ & 1.200 & 0.990 & $-4.94 \pm 0.07$ & -5.49 & $-6.60 \pm 0.19$ \\
\hline HD 117207 & G8IV/V & $0.724 \pm 0.002$ & 3.780 & 2.060 & $-5.10 \pm 0.02$ & -5.47 & \\
\hline HD 128311 & K0 & $0.973 \pm 0.004$ & $1.099 \pm 0.04$ & $2.180 \pm 0.02$ & $-4.42 \pm 0.02$ & $-4.54 \pm 0.06$ & $-4.54 \pm 0.21$ \\
\hline HD 130322 & K0III & $0.781 \pm 0.002$ & 0.880 & 1.080 & $-4.80 \pm 0.04$ & $-5.69 \pm 0.07$ & $-5.66 \pm 0.16$ \\
\hline HD 134987 & G5V & $0.691 \pm 0.020$ & $0.810 \pm 0.02$ & $1.590 \pm 0.02$ & $-5.13 \pm 0.07$ & -5.78 & -5.78 \\
\hline HD 141937 & $\mathrm{G} 2 / \mathrm{G} 3 \mathrm{~V}$ & $0.628 \pm 0.002$ & 1.520 & 9.700 & $-4.94 \pm 0.01$ & -5.39 & \\
\hline HD 150706 & G0 & $0.607 \pm 0.005$ & 0.820 & 1.000 & $-4.61 \pm 0.03$ & $-4.68 \pm 0.04$ & $-4.68 \pm 0.19$ \\
\hline HD 154345 & G8V & $0.728 \pm 0.005$ & $4.190 \pm 0.26$ & $0.947 \pm 0.09$ & $-4.94 \pm 0.05$ & -5.59 & $-6.02 \pm 0.16$ \\
\hline HD 164922 & $\mathrm{~K} 0 \mathrm{~V}$ & $0.799 \pm 0.005$ & $2.110 \pm 0.13$ & $0.360 \pm 0.05$ & $-5.08 \pm 0.02$ & -5.49 & -6.60 \\
\hline HD 168443 & G5 & $0.724 \pm 0.014$ & $0.300 \pm 0.02$ & $8.020 \pm 0.65$ & $-5.17 \pm 0.06$ & -5.50 & \\
\hline HD 168746 & G5 & $0.713 \pm 0.015$ & 0.065 & 0.230 & $-5.08 \pm 0.05$ & -4.96 & \\
\hline HD 169830 & F8V & $0.517 \pm 0.004$ & 0.810 & 2.880 & $-5.02 \pm 0.02$ & $-6.10 \pm 0.04$ & \\
\hline HD 170469 & G5 & $0.677 \pm 0.014$ & 2.240 & 0.670 & $-5.12 \pm 0.05$ & -3.01 & \\
\hline HD 178911B & G1V & $0.643 \pm 0.007$ & 0.320 & $6.292 \pm 0.06$ & $-4.96 \pm 0.04$ & $-5.56 \pm 0.18$ & \\
\hline HD 179949 & F8V & $0.548 \pm 0.009$ & $0.045 \pm 0.00$ & $0.950 \pm 0.04$ & $-4.77 \pm 0.02$ & $-5.25 \pm 0.10$ & $-5.25 \pm 0.25$ \\
\hline HD 183263 & G2IV & $0.678 \pm 0.012$ & $1.510 \pm 0.09$ & $3.670 \pm 0.30$ & $-5.14 \pm 0.05$ & -4.72 & \\
\hline HD 187123 & G5 & $0.661 \pm 0.010$ & $0.043 \pm 0.00$ & $0.520 \pm 0.04$ & $-5.06 \pm 0.03$ & -6.40 & \\
\hline HD 188015 & G5IV & $0.727 \pm 0.010$ & 1.190 & 1.260 & $-5.08 \pm 0.06$ & -4.78 & \\
\hline HD 189733 & G5 & $0.932 \pm 0.008$ & $0.031 \pm 0.00$ & $1.150 \pm 0.04$ & -4.50 & $-4.74 \pm 0.14$ & $-4.91 \pm 0.19$ \\
\hline HD 190360 & $\mathrm{G} 6 \mathrm{IV}+\ldots$ & $0.749 \pm 0.001$ & $0.128 \pm 0.00$ & $0.057 \pm 0.02$ & $-5.13 \pm 0.02$ & -6.71 & $-7.45 \pm 0.21$ \\
\hline HD 192263 & K0 & $0.938 \pm 0.015$ & 0.150 & 0.720 & $-4.54 \pm 0.04$ & $-5.06 \pm 0.20$ & $-5.06 \pm 0.35$ \\
\hline HD 195019 & G3IV-V & $0.662 \pm 0.007$ & $0.139 \pm 0.01$ & $3.700 \pm 0.30$ & $-5.11 \pm 0.03$ & $-7.51 \pm 0.17$ & $-7.42 \pm 0.17$ \\
\hline HD 196885 & F8IV: & $0.559 \pm 0.006$ & $2.370 \pm 0.02$ & $2.580 \pm 0.16$ & -4.99 & -5.59 & \\
\hline HD 209458 & F8 & $0.594 \pm 0.015$ & $0.045 \pm 0.00$ & $0.690 \pm 0.02$ & $-5.01 \pm 0.04$ & $-6.77 \pm 0.20$ & \\
\hline HD 210277 & G0 & $0.773 \pm 0.006$ & $1.100 \pm 0.02$ & $1.230 \pm 0.03$ & $-5.09 \pm 0.03$ & -5.74 & -5.74 \\
\hline HD 216770 & K0V & $0.821 \pm 0.004$ & 0.460 & 0.650 & -4.94 & -4.95 & \\
\hline HD 217107 & G8IV & $0.744 \pm 0.006$ & $0.073 \pm 0.00$ & $1.330 \pm 0.05$ & $-5.12 \pm 0.02$ & $-6.15 \pm 0.16$ & -6.81 \\
\hline $14 \mathrm{Her}$ & K0V & $0.877 \pm 0.006$ & $2.770 \pm 0.05$ & $4.640 \pm 0.19$ & $-5.08 \pm 0.03$ & -6.13 & $-6.34 \pm 0.14$ \\
\hline
\end{tabular}


Table 1. continued.

\begin{tabular}{|c|c|c|c|c|c|c|c|}
\hline Star & ST & $(B-V)$ & $a_{\mathrm{pl}}$ & $M_{\mathrm{pl}}$ & $\log \left(R_{\mathrm{HK}}^{\prime}\right)$ & $\log \left(\frac{L_{\mathrm{X}}}{L_{\mathrm{bol}}}\right)^{a}$ & $\log \left(\frac{L_{\mathrm{X}}}{L_{\mathrm{bol}}}\right)^{b}$ \\
\hline 16 Cyg & G2V & $0.643 \pm 0.006$ & $1.680 \pm 0.03$ & $1.680 \pm 0.07$ & $-5.12 \pm 0.03$ & -5.90 & -7.33 \\
\hline $47 U M a$ & G0V & $0.624 \pm 0.003$ & $2.110 \pm 0.02$ & $2.600 \pm 0.07$ & $-5.04 \pm 0.02$ & $-6.66 \pm 0.04$ & $-7.39 \pm 0.21$ \\
\hline $51 \mathrm{Peg}$ & G5V & $0.666 \pm 0.007$ & 0.052 & $0.468 \pm 0.01$ & -5.11 & $-6.91 \pm 0.09$ & $-7.43 \pm 0.18$ \\
\hline $55 \mathrm{Cnc}$ & G8V & $0.869 \pm 0.012$ & $0.038 \pm 0.00$ & $0.024 \pm 0.00$ & $-5.06 \pm 0.04$ & $-6.37 \pm 0.11$ & $-6.32 \pm 0.14$ \\
\hline $70 \mathrm{Vir}$ & G5V & $0.714 \pm 0.007$ & 0.480 & 7.440 & -5.02 & $-6.64 \pm 0.13$ & $-6.64 \pm 0.28$ \\
\hline$\epsilon E r i$ & $\mathrm{~K} 2 \mathrm{~V}$ & $0.881 \pm 0.007$ & $3.390 \pm 0.36$ & $1.550 \pm 0.24$ & -4.52 & $-4.99 \pm 0.00$ & $-4.89 \pm 0.12$ \\
\hline$\rho C r B$ & $\mathrm{G} 2 \mathrm{~V}$ & $0.612 \pm 0.003$ & 0.220 & 1.040 & $-5.09 \pm 0.02$ & -6.13 & -6.13 \\
\hline$\tau$ Boo & F7V & $0.508 \pm 0.001$ & 0.046 & 3.900 & -4.67 & $-5.29 \pm 0.02$ & $-5.18 \pm 0.12$ \\
\hline$v$ And & F8V & $0.536 \pm 0.007$ & $0.059 \pm 0.00$ & $0.690 \pm 0.03$ & -5.00 & $-6.00 \pm 0.07$ & $-6.00 \pm 0.22$ \\
\hline
\end{tabular}

References. ${ }^{(a)}$ Kashyap et al. (2008); ${ }^{(b)}$ Poppenhaeger et al. (2010).

Table 2. Stellar parameters and chromospheric and coronal activity indicators for the sample of stars without planets (Schmitt 1997).

\begin{tabular}{lcccc}
\hline \hline Star & ST & $(B-V)$ & $\log \left(R_{\mathrm{HK}}^{\prime}\right)$ & $\log \left(\frac{L_{\mathrm{X}}}{L_{\mathrm{bol}}}\right)$ \\
\hline HD 4614 & G0V... & $0.587 \pm 0.003$ & $-4.93 \pm 0.01$ & -6.59 \\
HD 10700 & G8V & $0.727 \pm 0.007$ & $-5.01 \pm 0.02$ & -6.55 \\
HD 13974 & G0V & $0.607 \pm 0.005$ & $-4.71 \pm 0.01$ & -5.13 \\
HD 14412 & G8V & $0.724 \pm 0.003$ & $-4.89 \pm 0.04$ & -6.13 \\
HD 14802 & G2V & $0.608 \pm 0.017$ & $-5.06 \pm 0.07$ & -5.06 \\
HD 16895 & F7V & $0.514 \pm 0.002$ & -4.92 & -6.12 \\
HD 19373 & G0V & $0.595 \pm 0.007$ & $-5.01 \pm 0.02$ & -6.83 \\
HD 30495 & G3V & $0.632 \pm 0.006$ & -4.61 & -4.75 \\
HD 30652 & F6V & $0.484 \pm 0.003$ & -4.60 & -5.03 \\
HD 69830 & K0V & $0.754 \pm 0.009$ & -4.98 & -5.85 \\
HD 72673 & K0V & $0.780 \pm 0.010$ & $-4.98 \pm 0.04$ & -5.84 \\
HD 90839 & F8V & $0.541 \pm 0.008$ & -4.83 & -5.53 \\
HD 101501 & G8Vvar & $0.723 \pm 0.014$ & -4.57 & -5.04 \\
HD 102870 & F8V & $0.518 \pm 0.015$ & -4.90 & -5.85 \\
HD 103095 & G8Vp & $0.754 \pm 0.009$ & $-4.88 \pm 0.02$ & -6.48 \\
HD 104304 & K0IV & $0.760 \pm 0.021$ & -4.95 & -6.09 \\
HD 109358 & G0V & $0.588 \pm 0.009$ & $-4.92 \pm 0.03$ & -6.44 \\
HD 114710 & G0V & $0.572 \pm 0.006$ & -4.75 & -5.58 \\
HD 115617 & G5V & $0.709 \pm 0.007$ & -5.07 & -6.53 \\
HD 117176 & G5V & $0.714 \pm 0.007$ & -5.02 & -7.66 \\
HD 131156 & G8V+... & $0.720 \pm 0.015$ & -4.37 & -4.62 \\
HD 141004 & G0Vvar & $0.604 \pm 0.008$ & $-4.98 \pm 0.02$ & -6.05 \\
HD 142860 & F6V & $0.478 \pm 0.006$ & -4.76 & -6.49 \\
HD 144579 & G8V & $0.734 \pm 0.001$ & $-5.00 \pm 0.01$ & -6.19 \\
HD 161797 & G5IV & $0.750 \pm 0.015$ & $-5.15 \pm 0.06$ & -6.32 \\
HD 185144 & K0V & $0.786 \pm 0.007$ & $-4.88 \pm 0.06$ & -5.81 \\
\hline
\end{tabular}

small outermost planets, a result corroborating the one obtained by Poppenhaeger et al. (2010) for the relationship between $\mathrm{X}$-ray luminosities and planetary parameters. In this context, the Spearman coefficients from the correlation study of $\log \left(R_{\mathrm{HK}}^{\prime}\right)$ with $\log \left(\frac{L_{\mathrm{X}}}{L_{\mathrm{bol}}}\right)$, also displayed in Table 3 , show a significant correlation between chromospheric and coronal activity indicators for stars with close-in planets. This correlation indicates that the relations found by Poppenhaeger et al. (2010) between the coronal activity indicator and the planetary parameters must be the same as in our analysis of stellar chromospheric activity and the presence of planets around a star.

As an additional statistical test to verify that the present data sets, segregated by planetary distances, are signiPcantly different from one another, we applied the Kolmogorov-Smirnov test (Press et al. 1992), which calculates the probability that two distributions are derived from the same parent distribution. According to the K-S test, the $p$ value indicates the probability that both distributions come from the same origin. We conducted

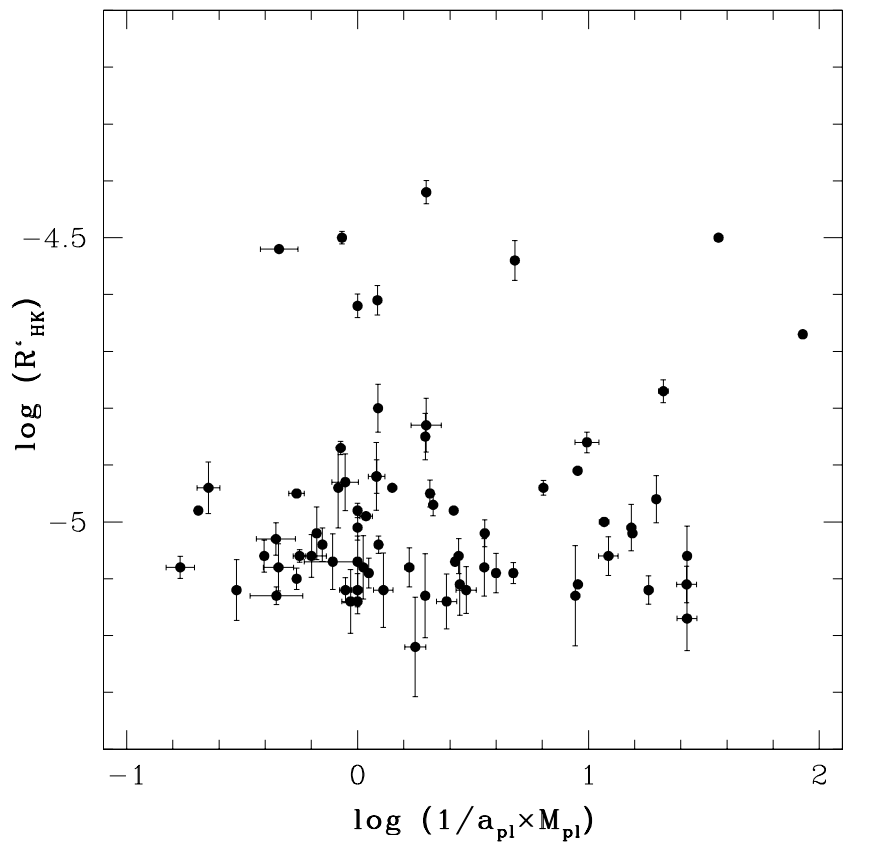

Fig. 2. Chromospheric activity indicator $\log \left(R_{\mathrm{HK}}^{\prime}\right)$ as a function of the product of the planetary mass with the reciprocal semi-major axis $\log \left(1 / a_{\mathrm{pl}} \times M_{\mathrm{pl}}\right)$ for our sample of stars with planets.

a K-S analysis, considering the $\log \left(R_{\mathrm{HK}}^{\prime}\right)$ of stars with planets within $a_{\mathrm{pl}} \leq 0.20 \mathrm{AU}(20$ stars $)$ and stars with planets beyond $a_{\mathrm{pl}} \geq 0.50 \mathrm{AU}$ (43 stars), once again following the strategy adopted by Poppenhaeger et al. (2010). Figure 3 shows the cumulative functions for both $\log \left(R_{\mathrm{HK}}^{\prime}\right)$ distributions. The errors were calculated considering the errors in $\log \left(R_{H K}^{\prime}\right)$ by applying random fluctuations with Gaussian distribution. The final values of $D$ are the average of the standard deviation after the application of the fluctuations. The probability value of about $53 \%$ obtained on the K-S test is consistent with the two distributions drawn from the same population. In agreement with Poppenhaeger et al. (2010) in their analysis of coronal X-ray emission, the present result indicates that both distributions of chromospheric activity indicator $\log \left(R_{\mathrm{HK}}^{\prime}\right)$ of stars with close-in and far-out planets are very similar.

\subsection{Comparison of chromospheric activity indicator in stars with and without planets}

In a search for systematic differences, we compared the behavior of chromospheric activity indicator $\log \left(R_{\mathrm{HK}}^{\prime}\right)$ in stars with 
Table 3. Statistical analysis of chromospheric activity indicator $\log \left(R_{\mathrm{HK}}^{\prime}\right)$ and planetary parameters and between coronal activity indicator $\log \left(\frac{L_{\mathrm{X}}}{L_{\mathrm{bol}}}\right)$ for stars with planets with semi-major axis $a_{\mathrm{pl}} \leq 0.2 \mathrm{AU}$.

\begin{tabular}{lcccc}
\hline \hline Parameters & $\begin{array}{c}\text { Spearman's } \rho \\
\text { (Kashyap }\end{array}$ & $\begin{array}{c}\text { Probability } p \\
\text { al. 2009) }\end{array}$ & $\begin{array}{c}\text { Spearman's } \rho \\
\text { (Poppenhaeger et al. 2010) }\end{array}$ & $\begin{array}{c}\text { Probability } p \\
\text { (Ko } 20.30 \pm 0.12\end{array}$ \\
\hline $\log R_{\mathrm{HK}}^{\prime}$ with $\log a_{\mathrm{pl}}$ & $-0.26 \pm 0.06$ & $0.30 \pm 0.06$ & $0.34 \pm 0.10$ \\
$\log R_{\mathrm{HK}}^{\prime}$ with $\log M_{\mathrm{pl}}$ & $0.13 \pm 0.07$ & $0.61 \pm 0.19$ & $0.20 \pm 0.08$ & $0.52 \pm 0.16$ \\
$\log R_{\mathrm{HK}}^{\prime}$ with $\log 1 / a_{\mathrm{pl}} \times M_{\mathrm{pl}}$ & $0.17 \pm 0.06$ & $0.51 \pm 0.17$ & $0.32 \pm 0.06$ & $0.29 \pm 0.09$ \\
$\log R_{\mathrm{HK}}^{\prime}$ with $\log \frac{L_{\mathrm{X}}}{L_{\mathrm{bol}}}$ & $0.64 \pm 0.09$ & $0.008 \pm 0.015$ & $0.92 \pm 0.04$ & $5.0 \times 10^{-5} \pm 2 \times 10^{-4}$ \\
\hline
\end{tabular}

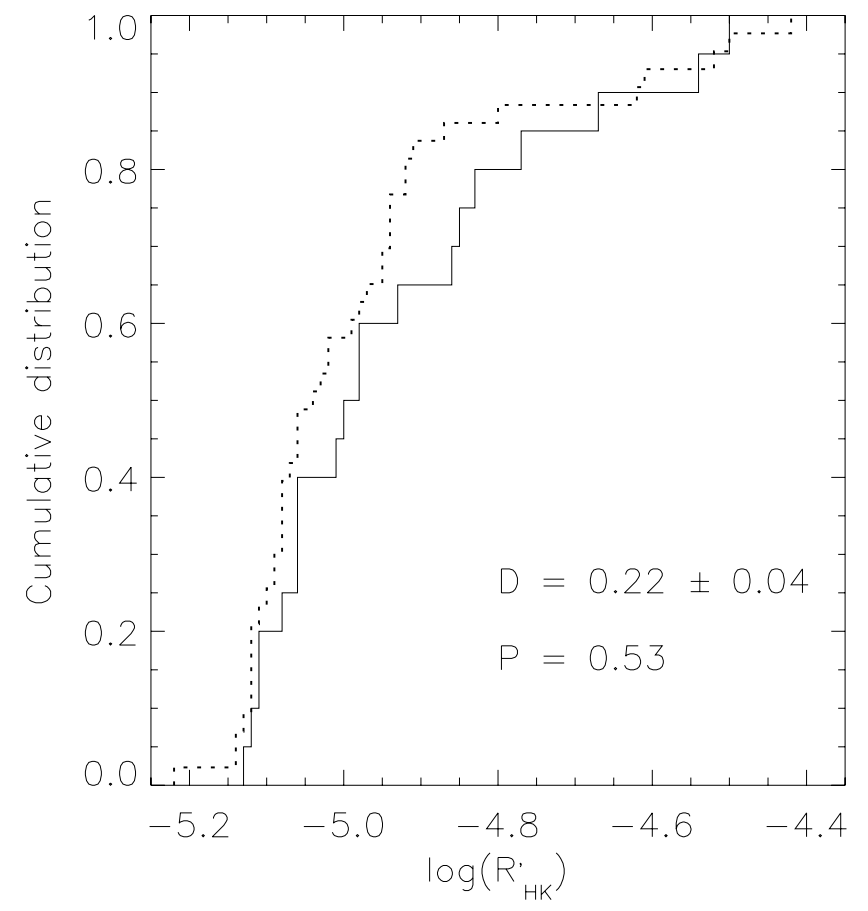

Fig. 3. The cumulative distributions of $\log \left(R_{\mathrm{HK}}^{\prime}\right)$ for stars with $a_{\mathrm{pl}} \leq$ $0.2 \mathrm{AU}$ (solid curve) and $a_{\mathrm{pl}} \geq 0.5 \mathrm{AU}$ (dashed curve).

and without detected planets. In Fig. 4 we show $\log \left(R_{\mathrm{HK}}^{\prime}\right)$ versus color index $(B-V)$ for both samples of stars, with no discernible differences. Both samples tend to follow the well known behavior of $\log \left(R_{\mathrm{HK}}^{\prime}\right)$, characterized by a decrease in chromospheric activity with increasing color index. In addition, in order to check for systematic differences between stars with and without detected planets, we compared the distributions of $\log \left(R_{\mathrm{HK}}^{\prime}\right)$ for the two aforementioned samples, considering only stars hosting close-in planets, in other words, stars with planets with $a_{\mathrm{pl}} \leq 0.20$ AU. Figure 5 shows the cumulative functions for both distributions. A K-S test reveals that the probability of both samples being drawn from the same parent distribution is $27 \%$, reinforcing the previous scenario, which shows no clear evidence of enhanced chromospheric activity associated to the presence of planetary companions.

\section{Conclusions}

We analyzed a sample of 74 stars with planets in the solar neighborhood and present chromospheric activity indicator $\log \left(R_{\mathrm{HK}}^{\prime}\right)$, searching for possible effects of star-planet interaction on the stellar chromosphere. From these analyses, we found no signiPcant correlations between the chromospheric activity indicator $\log \left(R_{\mathrm{HK}}^{\prime}\right)$ and planetary parameters: semi-major axis and product of the planetary mass with the reciprocal

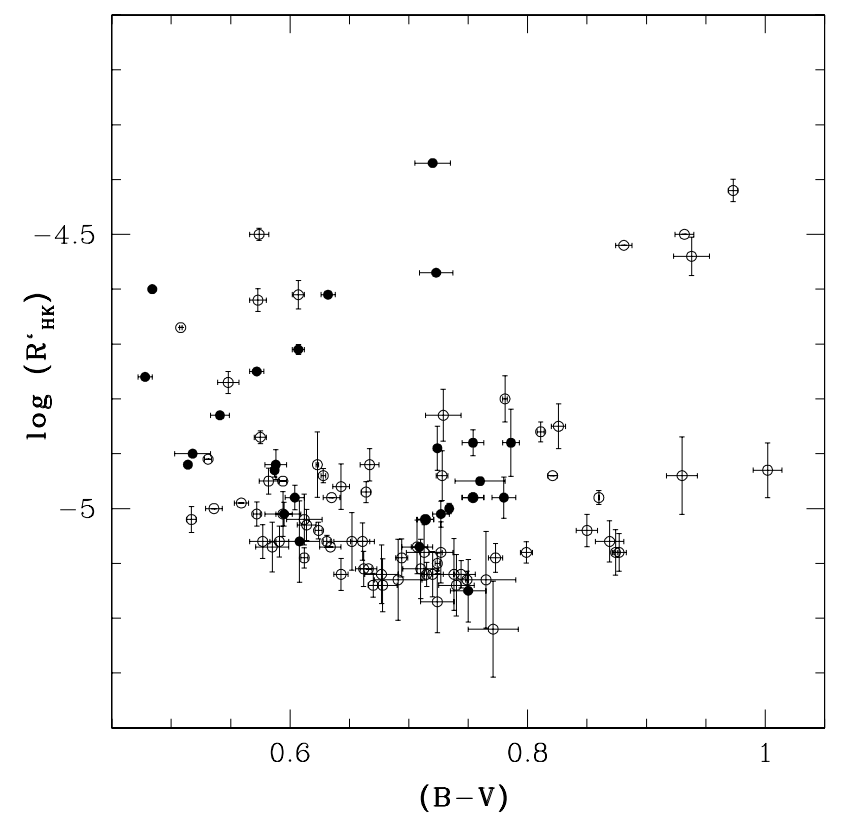

Fig. 4. Chromospheric activity indicator $\log \left(R_{\mathrm{HK}}^{\prime}\right)$ as a function of color index $(B-V)$. Open circles denote stars with planets and solid circles stars without planets.

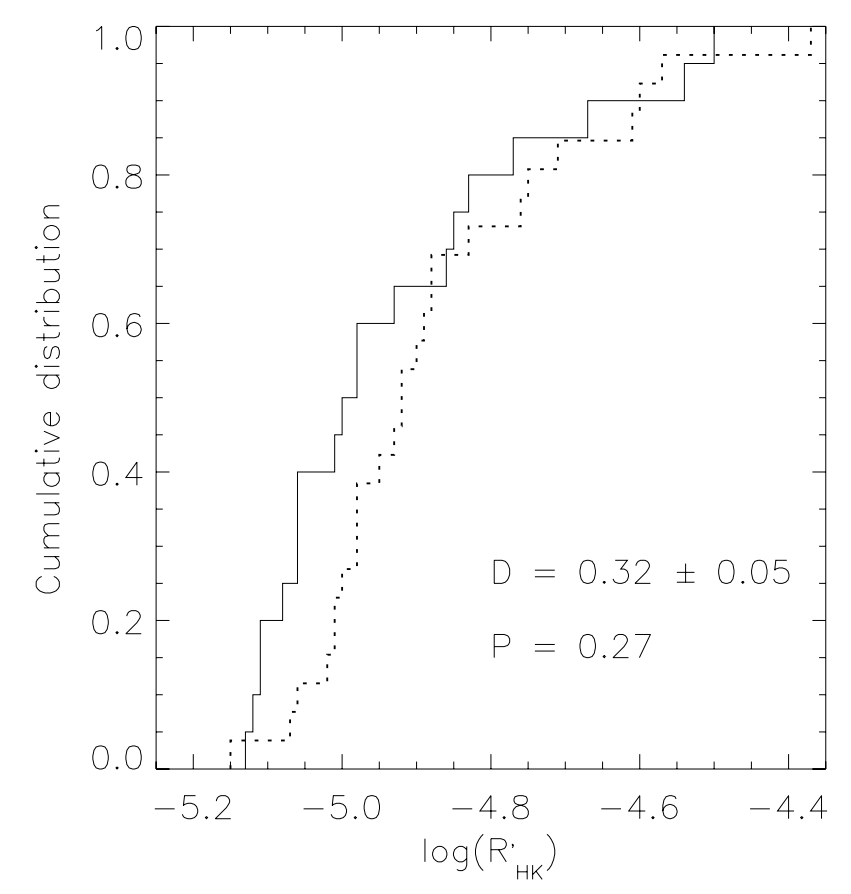

Fig. 5. The cumulative distributions of $\log \left(R_{\mathrm{HK}}^{\prime}\right)$ for stars with planets (solid curve) and stars without planets (dashed curve). For the sample of stars hosting planets, we are only considering stars with planets with $a_{\mathrm{pl}} \leq 0.20 \mathrm{AU}$. 
semi-major axis. However, we found a possible correlation between the $\log \left(R_{\mathrm{HK}}^{\prime}\right)$ and mass and between the product of planetary mass and reciprocal semi-major axis, indicating that massive close-in planets are often found around stars with an enhanced chromospheric activity indicator. Such a result supports the one obtained by Poppenhaeger et al. (2010) in their analysis of X-ray coronal luminosity in stars with planets. According to these authors, this dependence can be ascribed to selection effects, since the Doppler method for planet detection favors small and far-out planets around stars with low activity. Indeed, the present analysis shows a strong correlation between chromospheric and coronal activity indicators of stars with planets. Additionally, a statistical comparison between the $\log \left(R_{\mathrm{HK}}^{\prime}\right)$ indicator in stars with and without detected planets shows no clear evidence of enhanced chromospheric activity associated with planetary companions. In summary, our analysis reveals no clear evidence of enhanced planet-induced activity in the chromosphere of the stars. In agreement with the conclusions drawn by Poppenhaeger et al. (2010) in their analysis of the X-ray luminosity behavior of stars with planets, any trends observed in the present study seem to be mostly the result of selection effects.

Acknowledgements. Research activities at the Stellar Board of Universidade Federal do Rio Grande do Norte are supported by continuous grants from the Brazilian agencies CNPq and FAPERN (J. R. De Medeiros and B. L. Canto Martins). M. L. Chagas and S. Alves acknowledge graduate fellowships from the CAPES Brazilian agency. L. P. de Souza Neto and I. C. Leão acknowledge fellowships of the CNPq Brazilian agency. We warmly thank the anonymous referee for a careful reading and for suggestions that largely improved this paper.

\section{References}

Alves, S., do Nascimento, J. D., Jr, \& de Medeiros, J. R. 2010, MNRAS, 408, 1770

Butler, R. P., \& Marcy, G. W. 1996, ApJ, 464, 153

de Medeiros, J. R., Setiawan, J., Hatzes, A. P., et al. 2009, A\&A, 504, 617

ESA 1997, The Hipparcos and Tycho Catalogues, ESA SP-1200

Fares, R., Donati, J.-F., Moutou, C., et al. 2010, MNRAS, 406, 409

Flower, P. J. 1996, ApJ, 469, 355

Jetsu, L., \& Pelt, J. 1996, A\&AS, 118, 58

Kashyap, V. L., Drake, J. J., \& Saar, S. H. 2008, ApJ, 687, 1339

Konacki, M., Torres, G., Jha, S., \& Sasselov, D. D. 2003, Nature, 421, 507

Konacki, M., Torres, G., Sasselov, D. D., et al. 2004, ApJ, 609, 37

Marcy, G. W., Butler, R. P., Fischer, D., et al. 2001, ApJ, 556, 296

Mayor, M., \& Queloz, D. 1995, Nature, 378, 355

Noyes, R. W., Hartmann, L. W., Baliunas, S. L., Duncan, D. K., \& Vaughan, A. H. 1984, ApJ, 279, 763

Press, W. H., Teukolsky, S. A., Vetterling, W. T., \& Flannery, B. P. 1992, Numerical Recipes in FORTRAN (Cambridge: Cambridge Univ. Press) Poppenhaeger, K., Robrade, J., \& Schmitt, J. H. M. M. 2010, A\&A, 515, A98 Schmitt, J. H. M. M. 1997, A\&A, 318, 215

Schneider, J. 2010, The Extrasolar Planets Encyclopaedia, Web Resource: http://exoplanet.eu

Shkolnik, E., Walker, G. A. H., \& Bohlender, D. A. 2003, ApJ, 597, 1092

Shkolnik, E., Bohlender, D. A., Walker, G. A. H., \& Collier Cameron, A. 2008, ApJ, 676, 628

Wright, J. T., Marcy, G. W., Butler, R. P., \& Vogt, S. S. 2004, ApJS, 152, 261 